\title{
Effect of Acupuncture Administered in a Group Setting on Pain and Subjective Peripheral Neuropathy in Persons with Human Immunodeficiency Virus Disease
}

\author{
KENNETH D. PHILLIPS, Ph.D., ${ }^{1}$ WILLIAM D. SKELTON, Dipl.Ac., ${ }^{1,2}$ \\ and GREGORY A. HAND, Ph.D. ${ }^{1}$
}

\begin{abstract}
Objectives: The present study was performed to determine the effect of 5 weeks of acupuncture treatment in a group setting on pain and symptoms of peripheral neuropathy in human immunodeficiency virus (HIV)infected individuals.

Design: Twenty-one (21) subjects completed the study that consisted of a pretreatment and post-treatment case series design. The subjects completed the Pain Rating Scale and the Subjective Peripheral Neuropathy Screen (SPNS) before and after 5 weeks of acupuncture. The acupuncture treatments occurred two evenings per week. Each of the 10 sessions consisted of participants receiving 10-15 needle insertions in acupoints that addressed the individual's changing pattern of pain, sleep problems, or other health issues. The treatment utilized only main or common points located below the elbows and knees, and on the head, neck, and ears. Only reactive points were used in the acupuncture treatments. Needles were left in situ for 30-45 minutes.

Results: Comparison of the pretreatment and post-treatment Pain Rating Scale results indicated a significant reduction in present pain $(p=0.0002)$, least and most pain in the last 24 hours $(p<0.0001$ and $p=0.0004$, respectively) and the total pain summary score $(p<0.0001)$. Symptoms reported in the SPNS were reduced during the 5 weeks of acupuncture. Scores for pain/aching/burning, pins and needles, and numbness in the hands and feet were reduced (all significant at less than $p=0.0065)$, as well as the total summary score $(p<0.0001)$.

Conclusion: The results of this study indicate that subjective pain and symptoms of peripheral neuropathy were reduced during the period of individual acupuncture therapy delivered in a group setting. While the study design did not allow for control of nonspecific placebo factors, the data support the hypothesis that acupuncture in a group setting can reduce pain and neuropathic symptoms in HIV-infected individuals.
\end{abstract}

\section{INTRODUCTION}

$\mathbf{P}$ eripheral neuropathy can result from the direct infection of neurons with human immunodeficiency virus (HIV), opportunistic infection of neurons because of generalized immunosuppression, and from highly active antiretroviral therapy (HAART) (Hurst, 1999; Moyle and Sadler, 1998). Furthermore, the increased life expectancy of HIV-infected individuals through HAART increases the prevalence of pe- ripheral neuropathies (Sacktor, 2002). These sensory neuropathies are characterized by pain, numbness, tingling, and reduced functional capacity in the extremities. The most common is a distal sensory polyneuropathy presenting with painful, burning dysesthesia (Bouhassira et al., 1999).

Several studies have demonstrated the efficacy of acupuncture for treating chronic pain (Birch et al., 1996; Patel et al., Richardson and Vincent, Ter Riet et al., 1990). Those studies indicated that acupuncture is effective in re-

\footnotetext{
${ }^{1}$ The Mind-Body Research Group, University of South Carolina, Columbia, SC.

${ }^{2}$ The Acupuncture Clinic, Columbia, SC.
} 
ducing pain, with some studies showing a degree of pain relief in greater than $70 \%$ of individuals. It remains to be determined if the effect of acupuncture is mediated by physiologic or psychologic mechanisms, or a combination of the two.

While only a few studies have examined the effect of acupuncture on HIV-associated pain, the results are inconsistent (Greene et al., 1999; Patel et al., 1989; Shlay et al., 1998). However, the different findings might well result from varying methods among the studies. These differences, such as using either a standardized treatment or needling directed toward specific symptoms, would likely have a dramatic impact on the reported efficacy of acupuncture.

While a number of studies have examined the effect of acupuncture on pain, there is virtually no information on the therapeutic effect of acupuncture on symptoms of painless neuropathy. This general type of neuropathy, that is often reported by HIV-infected individuals, presents as "pins and needles" sensations or numbness in the limbs (Bouhassira et al., 1999).

The purpose of the present study was to determine the change in HIV-associated painful and nonpainful peripheral neuropathy during 5 weeks of acupuncture treatment. The intervention consisted of 10 treatments, two per week, with needling adjusted to specific symptoms reported by the subject. Painful and nonpainful symptoms of peripheral neuropathy were determined using subject rating scales before and after the 5-week treatment period.

\section{MATERIALS AND METHODS}

This study used a pretest/post-test pre-experimental design to examine whether acupuncture delivered in a group setting could improve subjective pain and symptoms of peripheral neuropathy in individuals with HIV/acquired immune deficiency syndrome (AIDS). A sample of 23 persons was recruited from an AIDS support organization and a private medical practice in Columbia, SC. Participants were HIV-seropositive and between the ages of 21 to 50 years. The participants were able to read and write English at a sixth grade level or above. Prospective participants were eligible to participate if they had not started, stopped, or changed doses of sedatives or analgesics within the last 30 days.

\section{Instruments}

Demographic data form. An 18-item demographic data form (DDF) was used to describe the sample. Specific questions addressed personal characteristics such as age, race, gender, education, relationship status, bed partners, income, route of infection, and medications. In addition, they were asked how often they suffered from sleep disturbances.

Pain rating scale. Pain intensity was measured using the Pain Rating Scale, a visual analogue scale with anchors rang- ing from 0 (no pain) to 10 (worst pain I have ever had). Participants were asked to rate their least pain in the last 24 hours, their worst pain in the last 24 hours, and their current pain. Regardless of the particular scale used, subjective ratings of pain intensity are considered valid (McGuire, 1992). Test-retest reliability $(r=0.95, p<0.001)$ has been demonstrated for visual analogues scales that measure pain intensity (Revill et al., 1976). Visual analogue scales may be more reliable in measuring changes in an individual's experience of pain than in comparing pain experience across groups, making them popular in studies of treatment outcomes (McGuire, 1992). Littman et al. (1985) found a high degree of correlation ( $r=0.89$ to 0.93 ) between a visual analogue scale, verbal descriptor scale, and a verbal pain relief scale. Reliability of visual analogue scales may be better to measure chronic pain, in better educated individuals who do not have impaired motor coordination (McGuire, 1992). Composites of 0 to 10 ratings, like the one described, maximizes reliability in clinical settings where detecting changes of pain intensity in individuals is needed (Jensen et al., 1999).

Subjective Peripheral Neuropathy Screen. Symptoms of peripheral neuropathy were measured using the Subjective Peripheral Neuropathy Screen (SPNS) (McArthur, 1998). This instrument is a brief self-report tool that was designed as a screening instrument for painful or nonpainful sensory neuropathy in HIV-infected subjects. The SPNS has been shown to be internally consistent (Cronbach $\alpha=0.86$ ). The test can discriminate for symptoms of paraesthesias, numbness, and lower extremity pain $(p<0.05)$. Further, it has been demonstrated that the SPNS assess differences in symptom severity $(p<0.05)$. Significant correlations were demonstrated for SPNS results and a neurologic examination, vibratory quantitative sensory testing, and severity measures. Sensitivity and specificity analysis indicates that numbness of the lower extremities is the symptom reported by the SPNS that most correctly classifies painful sensory neuropathy.

\section{Procedure}

The researchers conducted an information session at the AIDS support organization to describe acupuncture and the nature of the study. An announcement regarding the information session was mailed to all the clients of the AIDS support organization in their monthly newsletter. Forty-five (45) persons attended that information session. Individuals who were interested in participating in the study were asked to contact one of the researchers. At a subsequent meeting, potential participants were fully informed of the nature of the study and were asked to sign and return an informed consent sheet. Twenty-three (23) individuals were included in the study. The participants completed the pain and peripheral neuropathy scales on 3 consecutive days before and after completion of the acupuncture treatment. 
Table 1. Demographic Characteristics

\begin{tabular}{|c|c|c|}
\hline Characteristic & Frequency & Percent \\
\hline \multicolumn{3}{|l|}{ Gender } \\
\hline Male & 13 & 62 \\
\hline Female & 7 & 33 \\
\hline Transgendered & 1 & 5 \\
\hline \multicolumn{3}{|l|}{ Sexual Identity } \\
\hline Male homosexual & 10 & 48 \\
\hline Heterosexual & 8 & 38 \\
\hline Bisexual & 1 & 5 \\
\hline No response & 2 & 10 \\
\hline \multicolumn{3}{|l|}{ Race } \\
\hline Black & 10 & 48 \\
\hline White & 2 & 10 \\
\hline Other & 2 & 10 \\
\hline No response & 7 & 33 \\
\hline \multicolumn{3}{|l|}{ Education } \\
\hline Less than high school & 2 & 10 \\
\hline High school & 8 & 38 \\
\hline Some college & 7 & 33 \\
\hline College & 1 & 5 \\
\hline Graduate school & 2 & 10 \\
\hline \multicolumn{3}{|l|}{ Income Adequacy } \\
\hline Very adequate & 1 & 5 \\
\hline Adequate & 7 & 33 \\
\hline Inadequate & 9 & 43 \\
\hline Very inadequate & 4 & 19 \\
\hline \multicolumn{3}{|l|}{ Route of Infection } \\
\hline Sexual intercourse & 18 & 85 \\
\hline Transfusion & 1 & 5 \\
\hline Accidental exposure & 1 & 5 \\
\hline Other & 1 & 5 \\
\hline \multicolumn{3}{|l|}{ Health } \\
\hline Excellent & 2 & 10 \\
\hline Good & 6 & 29 \\
\hline Fair & 10 & 48 \\
\hline Poor & 3 & 14 \\
\hline
\end{tabular}

Intervention. The participants received acupuncture 2 evenings per week for 5 weeks, a total of 10 sessions. One of the researchers, a certified acupuncturist, performed acupuncture in a group session in a large room. Participants were seated in comfortable chairs in an ambient environment. Each participant had access to pillows to attain a comfortable position. Soft background music was played to facilitate relaxation. Needle insertion sites were prepared with alcohol before each treatment. Each patient received individualized acupuncture treatments that focused on specific needs and symptoms that the individual was experiencing. The rationale for this intervention was to test acupuncture as it is typically performed in practice. Point selection was based on the general principles of acupuncture and Traditional Chinese Medicine (East Asian Medical Studies Society, 1985). The treatment was modified over the course of the study to accommodate the individual's changing pattern of pain, sleep, or other health issues. Sterile, disposable acupuncture needles were used and guidelines of the Clean Needle Technique Manual for Acupuncturists (Brazil et al., 1977) were followed. The treatment utilized only main or common points located below the elbows and knees, and on the head, neck, and ears (Table 2). When treating body points, tenderness, twitching, tension, temperature, referred pain or other sensations determined if a point was used. Points that did not elicit one of these responses were not used.

Distal points were chosen whenever possible that would have additional benefits for specific pain or discomfort. Each treatment consisted of 10 or 15 needles that were left in situ for 30 to 45 minutes. Needles were manually manipulated briefly immediately after insertion. During the treatment, patients were encouraged to relax and to breathe deeply. The lighting of the room was dimmed slightly. Needles were removed, counted, and discarded in an appropriate container using universal precautions.

\section{Data analysis}

A graduate research assistant entered all data into a SAS 8.0 database (SAS Institute, Cary, NC). Data were verified by one of the researchers prior to any data analysis. Frequencies and percentages were calculated for the demographic variables. Means and standard deviations were calculated for the pretest and post-test for each of the three pain rating visual analogue scales, the total pain score, the individual items on the SPNS, and the total SPNS score. Paired $t$ tests were used to compare the mean pretest and posttest scores. To control multiple comparisons, the level of statistical significance was at $p<0.01$.

\section{RESULTS}

\section{Sample}

Twenty-three (23) subjects were enrolled to the study. One person died, and one person left the study prior to com-

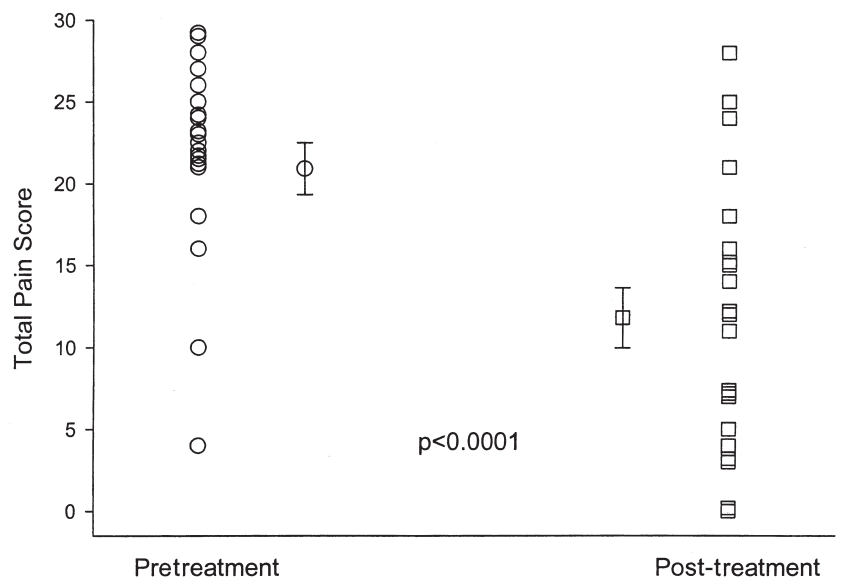

FIG. 1. Distribution of total pain scores among the individuals before (open circles) and after (open squares) 10 acupuncture sessions. The open circle with bars and open square with bars represent the mean and standard errors for pretreatment and post-treatment scores, respectively. The $p$ value represents the level of statistical significance between the mean pretreatment and posttreatment scores. 
Table 2. Acupuncture Points Used in This Study

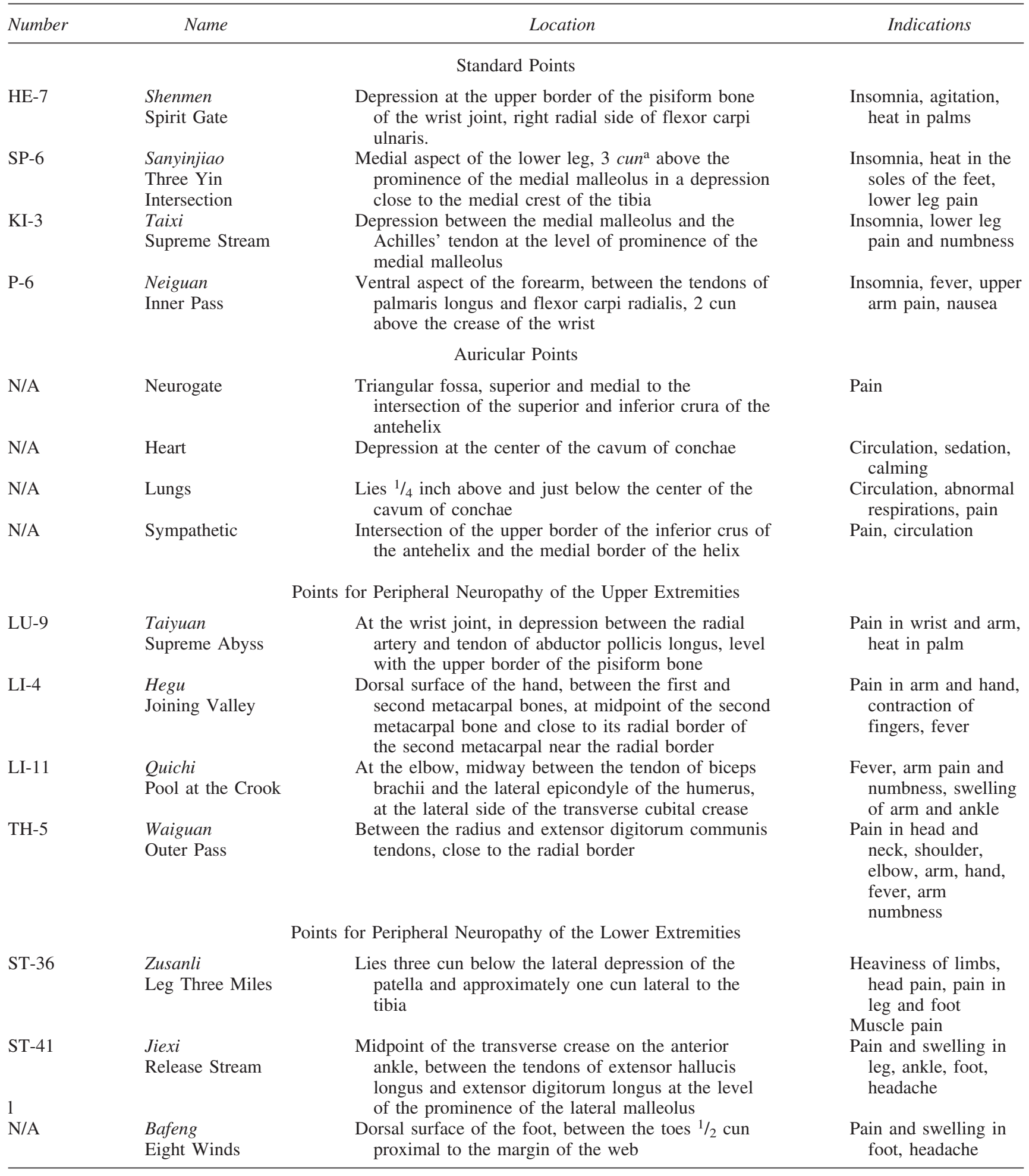

${ }^{a} \mathrm{Cun}$ is a unit of measurement equivalent to the width of the patient's interphalangeal joint. Three cun is the approximate width of the patient's hand. 
Table 3. Pain Rating Scale Individual Summary Scores

\begin{tabular}{lcccccrr}
\hline & \multicolumn{2}{c}{ Pretest } & & \multicolumn{2}{c}{ Post-test } & & \\
\cline { 2 - 3 } Variable & Mean & $S D$ & & Mean & & & \\
\hline Present pain & 6.9 & 2.7 & & 3.9 & 3.1 & 3.2 & 0.0002 \\
Least pain during the last 24 hours & 6.0 & 2.8 & & 3.0 & 2.3 & 5.1 & $<0.0001$ \\
Most pain during the last 24 hours & 8.0 & 2.3 & & 4.9 & 3.3 & 4.2 & 0.0004 \\
Total pain & 20.9 & 7.1 & & 11.8 & 8.3 & 5.0 & $<0.0001$ \\
\hline
\end{tabular}

$\mathrm{SD}$, standard deviation.

pletion because driving distance to the treatment site. The final sample consisted of $21 \mathrm{HIV}$-positive individuals who ranged in age from 29 to 50 (mean $=41.7$, standard deviation $[\mathrm{SD}]=6.6)$. Most $(n=11,52 \%)$ of the participants lived alone, and most $(n=17,81 \%)$ of them usually slept alone. The demographic characteristics of this sample are further described in Table 1 .

\section{Pain Rating Scale}

All scores related to pain showed significant reductions following acupuncture. As illustrated in Figure 1, the mean total pain score (the sum of the three questions related to pain) after the acupuncture treatment showed a significant reduction when compared to the pretreatment mean score. Pretreatment testing indicated that 18 subjects reported a total pain level above 20 with only 4 indicating total pain levels below 20. After acupuncture, only 4 subjects indicated a score above 20. Each of the individual pain questions also showed significant decreases when pre- and post-treatment scores were compared (Table 3 ). The changes in these scores ranged from the smallest decrease of $39 \%$ in "most pain during the last 24 hours" to the largest decrease of $50 \%$ in "least pain during the last 24 hours."

\section{Peripheral Neuropathy Scale}

All scores related to subjective peripheral neuropathy showed significant reductions following acupuncture. Figure 2 illustrates the individual and mean data points for total subjective peripheral neuropathy scores before and after the acupuncture treatment. Before acupuncture, 16 of the 21 subjects reported a score of greater than 25 (mean and standard error $[\mathrm{SE}]$ of $31.1 \pm 2.9$ ). After acupuncture, only 5 subjects reported a score greater than 25 (mean and $\mathrm{SE}$ of $15.7 \pm 2.8$ ). The responses for each of the individual questions related to peripheral neuropathy symptoms showed a reduction following acupuncture of at least $43 \%$ (pain, aching, burning in feet and legs) with the greatest reduction of $56 \%$ for questions related to "pain, aching, and burning in hands and arms" and "numbness in hands" (Table 4).

\section{DISCUSSION}

The present study was performed to determine the effect of 5 weeks of individualized acupuncture treatment, delivered in a group setting, on pain and symptoms of peripheral neuropathy associated with HIV infection. Significant findings indicate that acupuncture significantly reduced present pain, most and least pain in the past 24 hours, and total summary pain. In addition, the acupuncture regimen reduced pain/aching/burning and pins/needles/numbness in the upper and lower limbs. The total subjective peripheral neuropathy summary score was also reduced significantly by the treatment.

The incidence of neurologic disease and central nervous system infections associated with HIV infection has declined with the advent of HAART. However, as the life expectancy of HIV-infected individuals increases, the prevalence of peripheral neuropathies in individuals with HIV likely is increasing (Sacktor, 2002). This problem is exacerbated by the neuropathy that results from HAART in addition to HIV

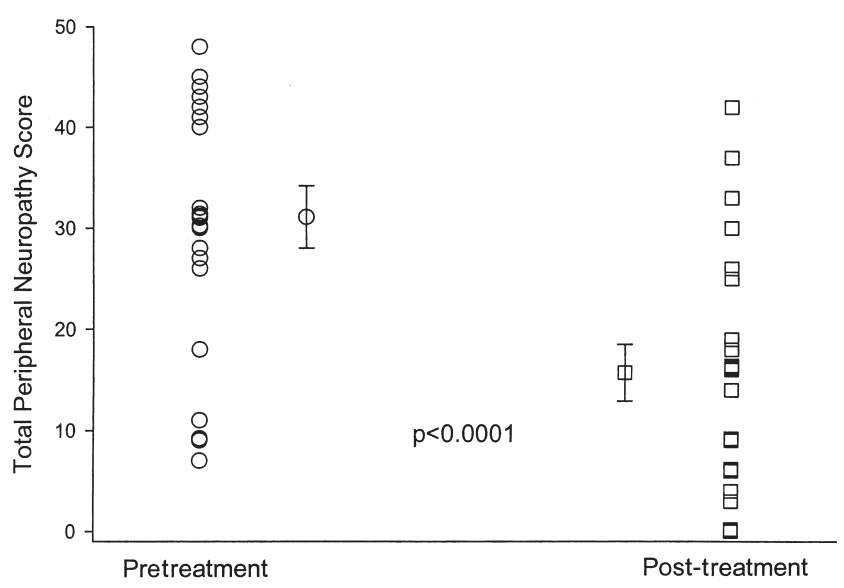

FIG. 2. Distribution of total subjective peripheral neuropathy scores among the individuals before (open circles) and after (open squares) 10 acupuncture sessions. The open circle with bars and open square with bars represent the mean and standard errors for pretreatment and post-treatment scores, respectively. The $p$ value represents the level of statistical significance between the mean pretreatment and post-treatment scores. 
Table 4. Subjective Peripheral Neuropathy Scale Individual Summary Scores

\begin{tabular}{|c|c|c|c|c|c|c|}
\hline \multirow[b]{2}{*}{ Variable } & \multicolumn{2}{|c|}{ Pretest } & \multicolumn{2}{|c|}{ Post-test } & \multirow[b]{2}{*}{$\mathrm{t}$ value } & \multirow[b]{2}{*}{$\mathrm{p}$ value } \\
\hline & Mean & $S D$ & Mean & $S D$ & & \\
\hline Pain, aching, burning in hands, arms & 3.6 & 2.7 & 1.6 & 1.7 & 4.2 & $<0.0001$ \\
\hline Pain, aching, burning in feet, legs & 7.2 & 2.5 & 4.1 & 3.1 & 4.1 & 0.0005 \\
\hline Pins and needles in hands, arms & 3.3 & 3.2 & 1.6 & 2.0 & 3.1 & 0.0063 \\
\hline Pins and needles in feet, legs & 5.8 & 3.4 & 3.0 & 3.3 & 3.7 & 0.0014 \\
\hline Numbness in hands, arms & 4.3 & 3.6 & 1.9 & 2.0 & 3.4 & 0.0031 \\
\hline Numbness in feet, legs & 6.8 & 2.7 & 3.4 & 3.2 & 4.4 & 0.0003 \\
\hline Total subjective peripheral neuropathy score & 31.1 & 13.0 & 15.7 & 12.6 & 4.7 & $<0.0001$ \\
\hline
\end{tabular}

$\mathrm{SD}$, standard deviation.

infection-related neuropathy and neuropathy resulting from opportunistic infections (Hurst, 1999; Moyle and Sadler, 1998; Patel et al., 1989). The HAART-related neuropathies generally result from administration of antiretrovirals such as zalcitabine, didanosine, or stavudine (Simpson and Tagliati, 1995). These sensory neuropathies, usually doserelated and primarily because of axonal toxicity, typically present as aching in the extremities; the most common is a distal sensory polyneuropathy with patients complaining of painful, burning dysesthesia. Because this neuropathy can result from HAART treatment as well as HIV infection, it does not improve dramatically with HAART-related reductions in HIV viral load (Simpson and Tagliati, 1995).

Several studies have demonstrated the efficacy of acupuncture for treating chronic pain (Birch et al., 1996; Richardson and Vincent, 1986). The meta-analysis by Patel et al. (1989) reported a rate of effect for acupuncture on pain syndromes as $70 \%$ for real acupuncture, $50 \%$ for sham acupuncture, and 30\% for control groups. Another meta-analysis (Ter Riet et al., 1990) identified 51 reports of acupuncture for chronic pain that included control group comparisons. While the studies included in the analysis varied considerably in terms of treatment regimen and type of control group, the overall results indicate that acupuncture produces some degree of pain relief. However, it is not clear whether the effect of acupuncture is mediated by physiologic or psychologic mechanisms, or a combination of the two.

Only a few studies have examined the effect of acupuncture on pain associated with HIV infection. A relatively large clinical trial (Shlay et al., 1998) examined the effects of a standardized regimen of acupuncture on pain related to peripheral neuropathy in HIV-infected subjects compared to a sham control group. The results indicated a small decline in pain scores at 6 and 14 weeks for both groups, with no significant difference between real and sham acupuncture. The results from the present study are markedly different from Shlay et al.'s (1998) findings in that we observed significant reductions in pain levels after 5 weeks of acupuncture. The greater decrease for pain scores in the present study could be due to the specificity of the acupuncture treatment for particular symptoms reported by the participants. The lack of significant treatment effect in Shlay and colleagues' study could be the result of the standardized acupuncture regimen "missing" the active or pertinent acupoints such that the study actually was comparing two sham treatments. This issue might be addressed by a future study utilizing both specific and standardized acupuncture procedures with either local anesthesia on the acupuncture sites, or naloxone administration to block the acupuncture-related effect of endogenous opiate release.

Peripheral sensory neuropathies associated with HIV disease may either be painful or painless (Bouhassira et al., 1999). Typical symptoms of painless neuropathy associated with HIV infection include "pins-and-needles" sensations or numbness in the limbs. Often, the sensations are in the upper or lower extremities, but not both. In the present study, the SPNS was used to examine the pins-and-needle sensations and numbness independently of pain/aching/burning. Our results indicated significant reductions in these non-pain variables of approximately 50\% during the acupuncture treatments. Bouhassira et al. (1999) demonstrated that the differential etiologies for painful and nonpainful neuropathies are poorly understood, there are clear delineations between the two groups of symptoms. In the present study, there was a high correlation between changes total pain scores and total peripheral neuropathy scores. This correlation is, in part, because all but two subjects reported a reduction in total pain during the acupuncture treatment. These two subjects were two of the five subjects who reported no change or increased total subjective peripheral neuropathy scores.

In summary, the present study determined the change in HIV-associated pain and peripheral neuropathy during 5 weeks of acupuncture treatment targeted at the participants' individual symptoms. Results indicate that during the acupuncture treatment period, present pain, most and least pain in the past 24 hours, and total summary pain was reduced significantly. Furthermore, the data show that sensations other than those associated with pain such as pins and needles and numbness in the upper and lower limbs were also reduced. The total subjective peripheral neuropathy 
summary score was reduced by approximately $50 \%$. The results of this study indicate that peripheral neuropathy and pain in HIV-infected individuals may be treated successfully through acupuncture directed at the individual's specific symptoms. Future research should include a randomized controlled trial of acupuncture for peripheral neuropathy directed at the subjects' individual symptomatology.

\section{ACKNOWLEDGMENTS}

This research was funded in part by Sponsored Programs and Research at the University of South Carolina. The authors gratefully acknowledge Jo Ann Harmon, R.N., Johnny M. Griffin, L.P.N., and Pamela Broadus, R.N., B.S.N., for their support in the completion of this work.

\section{REFERENCES}

Birch S, Hammerschlag R, Berman BM. Acupuncture in the treatment of pain. J Altern Complement Med 1996;2:101-124.

Bouhassira D, Attal N, Willer JC, Brasseur L. Painful and painless peripheral sensory neuropathies due to HIV infection: A comparison using quantitative sensory evaluation. Pain 1999;80:265-272.

Brazil J, Mitchell BB, Tu A, Skelton WD, eds. Clean Needle Technique Manual for Acupuncturists: Guidelines and Standards for the Clean and Safe Clinical Practice, 4th ed. Washington, DC: National Acupuncture Foundation, 1997.

East Asian Medical Studies Society. Fundamentals of Chinese Medicine. Brookline, MA: Paradigm, 1985.

Greene KB, Berger J, Reeves C, Moffat A, Standish LJ, Calabrese C. Most frequently used alternative and complementary therapies and activities by participants in the AMCOA study. J Assoc Nurses AIDS Care 1999;10:60-73.

Hurst M. Stavudine: An upate of its use in the treatment of HIV infection. Drugs 1999;58:919-949.

Jensen MP, Turner JA, Romano JM, Fisher LD. Comparative reliability and validity of chronic pain intensity measures. Pain 1999;83:157-162.

Littman GS, Walker BR, Schneider BE. Reassessment of verbal and visual analog ratings in analgesic studies. Clin Pharmacol Ther 1985;38:16-23.

McArthur JH. The reliability and validity of the subjective peripheral neuropathy screen. J Assoc Nurses AIDS Care 1998; 9:84-94.

McGuire DB. Comprehensive and multidimensional assessment and measurement of pain. J Pain Symptom Manage 1992;7: 312-319.

Moyle GJ, Sadler M. Peripheral neuropathy with nucleoside antiretrovirals-risk factors, incidence and management. Drug Safety 1998;19:481-494.

Patel M, Gutzwiller F, Paccaud F, Marazzi A. A meta-analysis of acupuncture for chronic pain. Int J Epidemiol 1989;18:900-906.

Revill SI, Robinson JO, Rosen M, Hogg MI. The reliability of a linear analogue for evaluating pain. Anaesthesia 1976;31: 1191-1198.

Richardson PH, Vincent CA. Acupuncture for the treatment of pain: A review of evaluative research. Pain 1986;24:15-40.

Sacktor N. The epidemiology of human immunodeficiency virusassociated neurological disease in the era of highly active antiretroviral therapy. J Neurovirol 2002;8(Suppl 2):115-121.

Shlay JC, Chaloner K, Max MB, Flaws B, Reichelderfer P, Wentworth D, Hillman S, Brizz B, Cohn DL. Acupuncture and amitriptyline for pain due to HIV-related peripheral neuropathy-a randomized controlled trial. JAMA 1998;280: $1590-1595$.

Simpson DM, Tagliati M. Nucleoside analogue-associated peripheral neuropathy in human immunodeficiency virus infection. J Acquir Immune Defic Syndr Hum Retrovirol 1995;9:153-161.

Ter Riet G, Kleijnen J, Knipschild P. Acupuncture and chronic pain: A criteria-based meta-analysis. J Clin Epidemiol 1990;43: 1191-1199.

\author{
Address reprint requests to: \\ Gregory A. Hand, Ph.D. \\ School of Public Health \\ University of South Carolina \\ 1300 Wheat Street \\ Columbia, SC 292908
}

E-mail: ghand@sc.edu 